\title{
Caractéristiques Physico-Chimiques Des Eaux De La Nappe Alluviale De La Vallée Du Goulbi N'Kaba Dans La Région De Maradi Au Niger
}

\author{
Laoualy Yahouza \\ Programme d'Hydraulique Rurale, Appui au Secteur Eau et Assainissement, \\ Niamey, Niger, Université Abdou Moumouni de Niamey, FAST, \\ Département de Géologie, Niamey, Niger \\ Sandao Issoufou \\ Université Abdou Moumouni de Niamey, FAST, \\ Département de Géologie, Niamey, Niger \\ Maman Sani Abdou Babaye \\ Université Dan Dicko Dan Koulodo, FST, \\ Département de Géologie, Maradi, Niger
}

Bruno Metral

Programme d'Hydraulique Rurale, Appui au Secteur Eau et Assainissement, Niamey, Niger

Boureima Ousmane

Université Abdou Moumouni de Niamey, FAST,

Département de Géologie, Niamey, Niger

Doi: 10.19044/esj.2018.v14n24p37～URL:http://dx.doi.org/10.19044/esj.2018.v14n24p37

\begin{abstract}
The Goulbi N'Kaba valley is located in the Maradi region of southcentral Niger. It contains an alluvial aquifer that is used for the drinking water supply, breeding, increasingly more irrigation and for the extractive industries. In addition, this valley is subject to the effects of climate change. A study is needed to improve the knowledge of this water resource, a condition for a better match between the uses and the potentialities of the aquifer. The investigations on the physicochemical parameters and the stable isotopes of the water molecule (oxygen 18 and deuterium), made it possible to reach the following main results: The waters of the alluvial aquifer are slightly acidic, with a $\mathrm{pH}$ ranging from 5,2 to 7.2 . The mineralization is relatively weak with conductivities between 89,3 and $325 \mu \mathrm{s} / \mathrm{cm}$, resulting in sodium and calcium bicarbonate facies. These waters are generally good for human consumption and for irrigation. However, in the center of the study area, fluorine and nitrate contents are observed that exceed the WHO guideline values (2006).
\end{abstract}


Keywords: Goulbi N'Kaba, alluvial aquifer, physicochemical parameters, irrigation.

\section{Résumé}

La vallée du Goulbi N'Kaba se situe dans la région de Maradi, au centre-sud du Niger. Elle renferme une nappe alluviale qui est sollicitée pour l'alimentation en eau potable, l'élevage, l'irrigation de plus en plus croissante et les industries extractives. Par ailleurs, cette vallée est soumise aux effets du changement climatique. Aussi, une étude s'avère nécessaire en vue d'améliorer la connaissance de cette ressource en eau, pour une meilleure adéquation entre les usages et les potentialités de l'aquifère. Les investigations portant sur les paramètres physicochimiques, ont permis d'atteindre les principaux résultats suivants : Les eaux de la nappe alluviale sont légèrement acides, avec un pH variant de 5,2 à 7,2. La minéralisation est relativement faible avec des conductivités comprises entre 89,3 et $325 \mu \mathrm{s} / \mathrm{cm}$, se traduisant par des faciès de type bicarbonatés sodiques et calciques. Ces eaux sont généralement bonnes pour la consommation humaine et pour l'irrigation. Cependant on observe au centre de la zone d'étude, des teneurs en fluor et en nitrates qui dépassent les valeurs guides de l'OMS (2006).

Mots clés: Goulbi N'Kaba, nappe alluviale, paramètres physico-chimiques, irrigation.

\section{Introduction}

La vallée du Goulbi N'Kaba en cours de fossilisation, traverse sur environ $270 \mathrm{~km}$, le centre de la région de Maradi et l'extrême nord-est de la région de Tahoua. Malgré le climat aride qui caractérise la zone (BEFACREP, 2009), la vallée du Goulbi N'Kaba renferme une nappe alluviale, sollicitée pour l'alimentation en eau potable, l'élevage, l'agriculture et les industries extractives. Les prélèvements en eau pour ces différents usages, sont de plus en plus croissants compte tenu du poids démographique (INS, 2012) et du développement de la petite irrigation (PIP, 2006), pratiquée pour pallier à l'insécurité alimentaire. Ainsi, ces ressources en eau, notamment celles de la nappe alluviale, constituent un enjeu majeur pour le développement socioéconomique de la région. L'objectif principal de cette étude, est d'améliorer les connaissances de cette nappe. Les objectifs spécifiques sont entre autres : la détermination et l'analyse des paramètres physico-chimiques des eaux de la nappe alluviale de la vallée du Goulbi N'Kaba.

\section{Cadre De L'etude}

La vallée du Goulbi N'Kaba prend sa source au Nigéria, traverse le centre de la région de Maradi et l'extrême sud-est de la région de Tahoua, 
avant de retourner au Nigéria. Elle se localise entre $13^{\circ} 20$ et $14^{\circ} 00$ de latitude Nord et $6^{\circ} 30$ et $8^{\circ} 10$ de longitude Est (fig.1).

Le climat de la zone d'étude, de type semi-aride, se caractérise par deux saisons bien distinctes, dont l'une sèche, de longue durée (8 à 9 mois) et l'autre humide de courte durée (juin à septembre). Les pluies sont de courtes durées et variables dans le temps et dans l'espace (Mahé et al., 2005). Pour la période de 1950 à 2015, les moyennes interannuelles sont respectivement de $525 \mathrm{~mm}$ à Maradi aéroport, $471 \mathrm{~mm}$ à Tessaoua et $368 \mathrm{~mm}$ à Dakoro. La température varie en moyenne de $18^{\circ} \mathrm{C}$ aux mois de décembre à mars, à 40,5 ${ }^{\circ} \mathrm{C}$ aux mois d'avril et mai. Les vents dominants sont, l'harmattan en saison sèche et la mousson en saison humide. L'évapotranspiration potentielle moyenne annuelle peut atteindre plus de $2000 \mathrm{~mm}$.
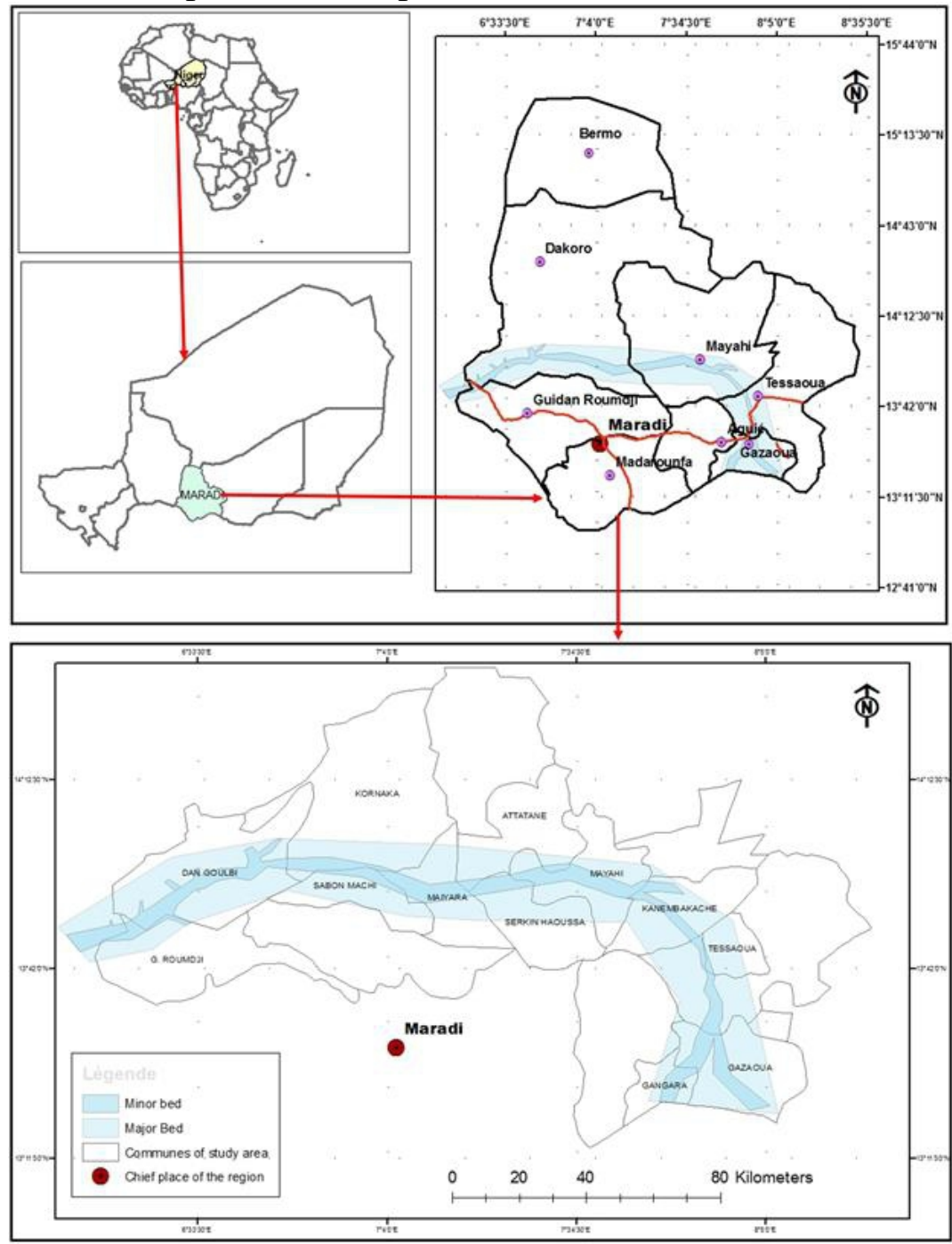

Fig. 1 : Cadre géographique de la zone d'étude 
Le réseau hydrographique, constitué essentiellement par le Goulbi N'Kaba et ses affluents, est en cours de fossilisation. Néanmoins, deux de ses affluents, prenant leur source au Nigéria (le Goulbi El Fadama et le Goulbi May Farou), ont des écoulements intermittents pendant la saison des pluies (SOGHETA, 1964). On y trouve également, quelques mares temporaires et semi-permanentes, localisées principalement dans la vallée.

Sur le plan géologique (fig.2), le remplissage du bassin de Goulbi N'Kaba est essentiellement constitué de sables grossiers et moyens à galets, provenant de l'altération du socle cristallin, du remaniement des grès du Continental Intercalaire, du continental Hamadien $(\mathrm{CI} / \mathrm{CH})$, et des alluvions anciennes. Les dépôts de comblement de la vallée, d'une épaisseur variant de 25 à 40 mètres (SOGHETA, 1964 ; Greigert, 1969) sont surmontés par de sables éoliens. Ces formations géologiques renferment un aquifère à nappe libre, capté à des profondeurs variant de $5 \mathrm{~m}$ dans le secteur amont de la zone d'étude, d'environ $30 \mathrm{~m}$ dans le secteur central et de moins de $20 \mathrm{~m}$ dans le secteur aval (Cabinet Labaran, 2015). Les débits d'exploitation sont plus importants dans les secteurs amont et aval où la nappe alluviale est moins profonde (SOGHETA, 1964 ; MHE, 1993). Les conductivités des eaux varient de 89,3 à plus de $325 \mu \mathrm{s} / \mathrm{cm}$. Le pH est légèrement acide mais tend à devenir neutre dans le secteur aval. Les températures des eaux de la nappe alluviale tournent au tour de $30^{\circ} \mathrm{C}$.

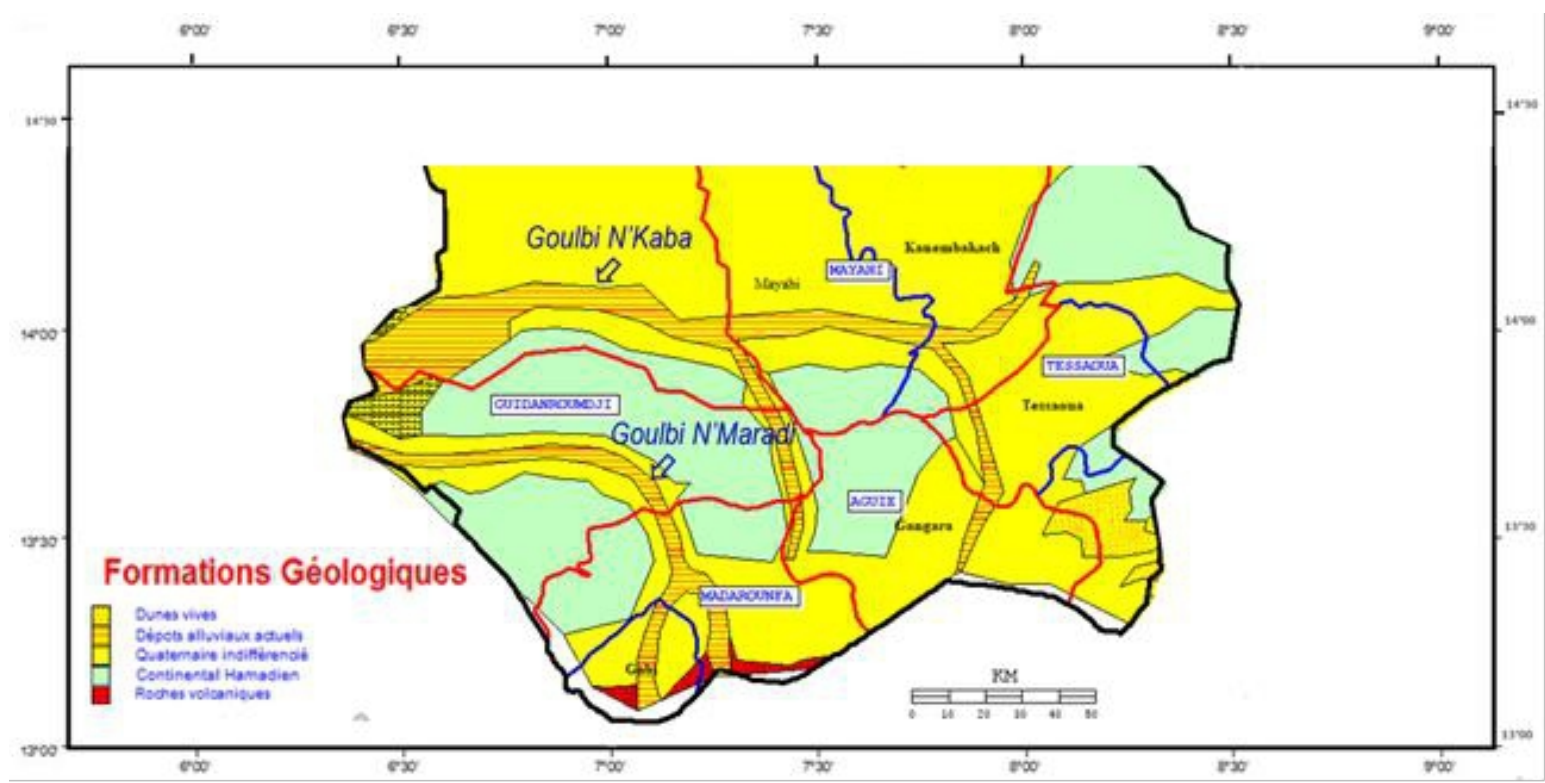

Fig. 2 : Carte modifiée et simplifiée des formations géologiques de la Région de Maradi (source : Base de données IRH-SIG-NER/MHA)

\section{Materiel Et Methodes}

Le matériel utilisé, comprend des données et des outils : 
○ Données : il s’agit de divers documents dont des mémoires de thèses et de masters, des cartes topographiques, géologiques, hydrogéologiques et des imageries satellitaires ; des paramètres physico-chimiques.

○ Outils : divers logiciels (Excel et Word, Diagramme, XLSTAT, Google Earth), un GPS, un appareil photo-numérique, des flacons, des glacières. Ils comprennent également des équipements de laboratoire pour les analyses physico-chimiques.

L'approche méthodologique est la suivante :

\section{○ Sélection des points d'eau échantillonnés}

23 échantillons d'eau des forages et des puits répartis judicieusement sur toute la zone d'étude ont été analysés (fig.3). Les paramètres physiques ont été mesurés in-situ et au laboratoire.

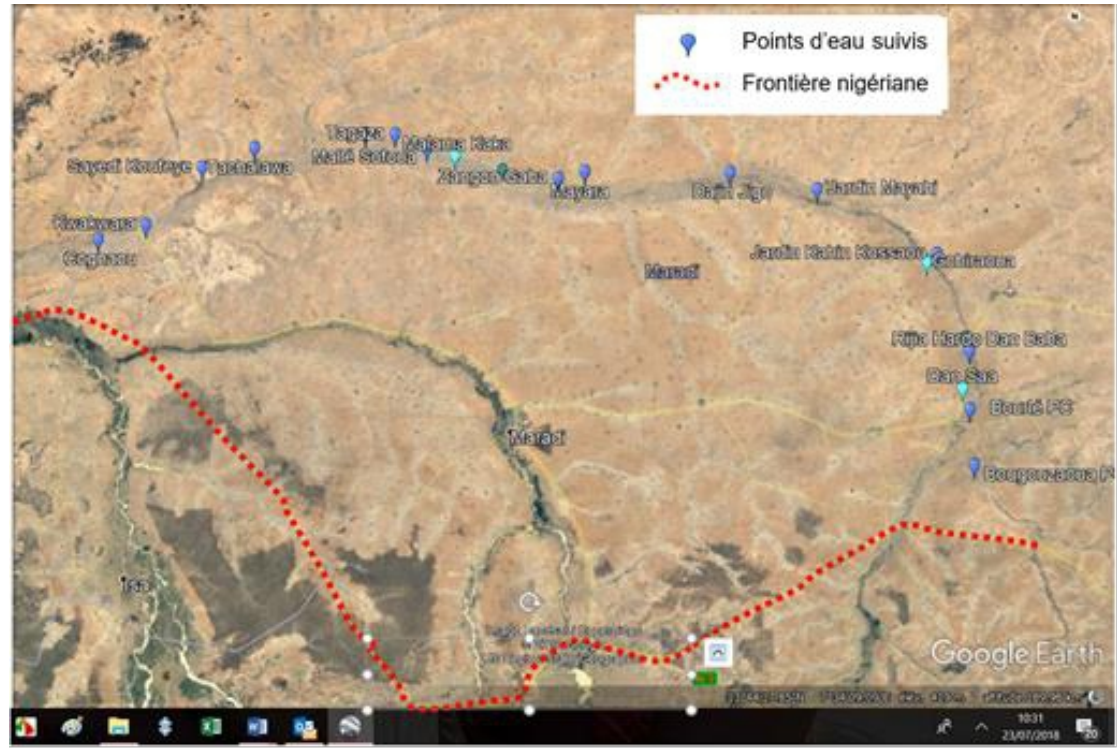

Fig. 3 : Localisation des ouvrages suivis (image Google Earth)

\section{$\bigcirc \quad$ Techniques analytiques :}

Les analyses ont été faites au laboratoire de la DRH/A de Zinder, les techniques utilisées sont: la spectrophotométrie, pour le dosage des Nitrates $\left(\mathrm{NO}_{3}\right)$, nitrites $\left(\mathrm{NO}_{2-}\right)$, sulfates $\left(\mathrm{SO}_{4--}\right)$, fluor $(\mathrm{F}-)$, fer total ; la volumétrie pour les ions calcium $\left(\mathrm{Ca}^{2+}\right)$, magnésium $\left(\mathrm{Mg}^{2+}\right)$, bicarbonates $\left(\mathrm{HCO}_{3}\right)$ et les chlorures $(\mathrm{Cl}-)$; et enfin, la photométrie à flamme pour les ions potassium $\left(\mathrm{K}^{+}\right)$et sodium $\left(\mathrm{Na}^{+}\right)$.

○ Traitement de données :

- Concernant les paramètres physiques, il a été déterminé les gammes de variations, les valeurs moyennes, les écart-types et établi des cartes thématiques. 
- Pour les éléments chimiques, il a été défini la balance qui doit être inférieure ou égale à $6 \%$ pour considérer l'analyse acceptable. La comparaison des ions majeurs a permis d'évaluer les proportions en pourcentage des différents éléments chimiques, leurs répartitions en diagramme et de déterminer les formules ioniques des eaux. Les éléments majeurs ont été représentés sur le diagramme de Piper afin de déterminer les faciès chimiques des eaux. Les corrélations bi variables entre éléments et une analyse en composante principale (ACP) ont été réalisées. Les indices de saturation des eaux vis-à-vis de certains minéraux (calcite, aragonite, dolomite et gypse) ont été déterminés. L'aptitude des eaux à l'irrigation a été évaluée à partir des indes SAR et RSC et le diagramme de Wilcox.

\section{Resultats Et Discussions}

Analyse des paramètres physiques

Les températures des eaux de la nappe alluviale varient de $28,4^{\circ} \mathrm{C}$ à $33,6^{\circ} \mathrm{C}$, avec une moyenne de $31,8^{\circ} \mathrm{C}$, et un écart-type de 1,3 pour la campagne de basses eaux. Cette variation reste identique en période de hautes eaux. Les faibles températures sont généralement relevées dans le secteur amont où la nappe est peu profonde (5 à $15 \mathrm{~m}$ ). Dans les secteurs central et aval, les températures des eaux augmentent et se situent entre 32 et $33^{\circ} \mathrm{C}$. Cependant, ces températures, dans leur ensemble, reflètent celle de l'air atmosphérique et restent conformes à celles des eaux des nappes alluviales de la région (Sandao, 2013 ; Boukari, 2014).

Les $\mathrm{pH}$ des eaux varient de 5,2 à 7,1 Unités $\mathrm{pH}$, avec une moyenne de 6,3 unités $\mathrm{pH}$, et un écart-type de 0,5 pour la campagne de basses eaux. Ceuxci restent quasi stables en période des hautes eaux. Cependant, il est à relever que des faibles $\mathrm{pH}$ (de 5 à 6 unités $\mathrm{pH}$ ) ont été observés dans les secteurs amont et aval de la vallée. Ceci pourrait s'expliquer par le fait que dans ces secteurs, la nappe est en système ouvert. Ces secteurs correspondraient à la zone de recharge. En ce qui concerne le secteur amont, le pH des eaux tend vers les valeurs neutres, le milieu à tendance à devenir un système fermé.

Les conductivités de la nappe alluviale varient de 62 à $325 \mu \mathrm{s} / \mathrm{cm}$, avec une moyenne de 162,3 $\mu \mathrm{s} / \mathrm{cm}$, et un écart-type de 86,1 en basses eaux. Elles varient peu selon les saisons. Cependant, ces conductivités croissent d'amont en aval (fig. 4). Cela semble confirmer que le contact eau roche devient de plus en plus long suivant le sens de l'écoulement. Ces résultats confirment ceux observés dans la distribution du $\mathrm{pH}$ et dans une moindre mesure, ceux de la température. 


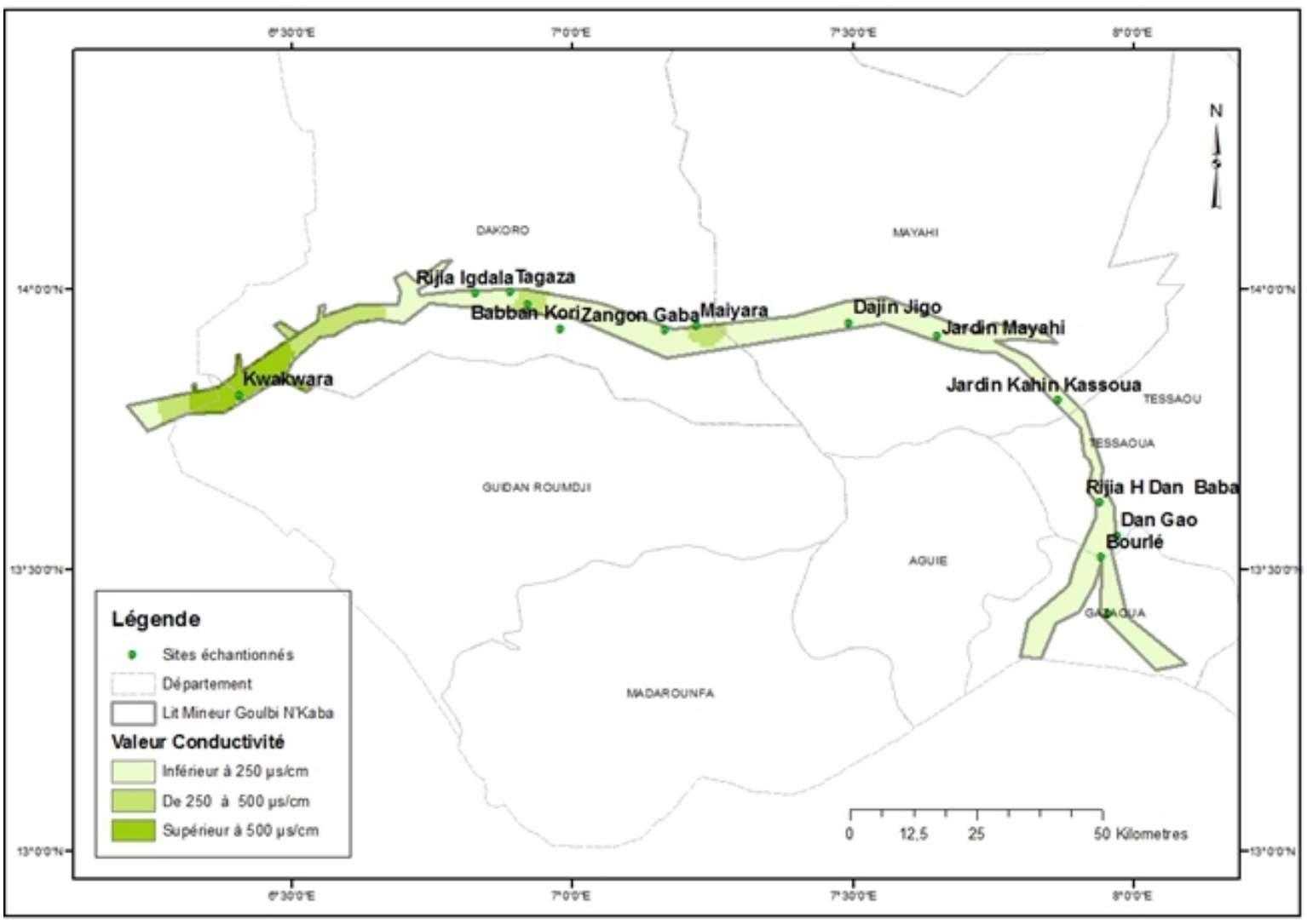

Fig. 4 : Répartition des valeurs de la conductivité en 2016, des eaux de la nappe alluviale

Analyse des paramètres chimiques

Les balances ioniques des résultats analytiques des eaux de la nappe alluviale sont dans l'intervalle $\pm 7 \%$. Ces résultats ont été considérés acceptables compte tenu des faibles concentrations ioniques, les rendant sensibles aux dosages volumétriques.

Les valeurs des concentrations ioniques des eaux de la nappe alluviale donnent les ordres d'abondance suivants : pour les anions, $\mathrm{HCO}_{3}>\mathrm{SO}_{4}>$ $\mathrm{CL}>\mathrm{NO}_{3}$ et pour les cations : $\mathrm{Na}>\mathrm{Ca}>\mathrm{Mg}>\mathrm{K}$

Les éléments chimiques des eaux de la nappe alluviale, représentés sur le diagramme de Piper (fig. 5), ont permis de déterminer les faciès suivants : le type bicarbonaté sodique, le type chloruré sodique et le type mixte (sulfaté - bicarbonaté et chloruré, calcique - sodique et magnésien). Cependant, ces faciès semblent évoluer de l'amont vers l'aval en fonction de la minéralisation totale. En effet, le faciès chloruré est observé dans le secteur amont où les eaux sont faiblement minéralisées, ce qui correspondrait à la zone de recharge potentielle. Le faciès est mixte au niveau du secteur central. Par contre, dans la zone aval où le système semble être fermé, la minéralisation plus élevée, on 
rencontre les faciès bicarbonatés sodiques et potassiques. Cela pourrait s'expliquer par un temps de séjour plus long et une recharge faible dans ce secteur.

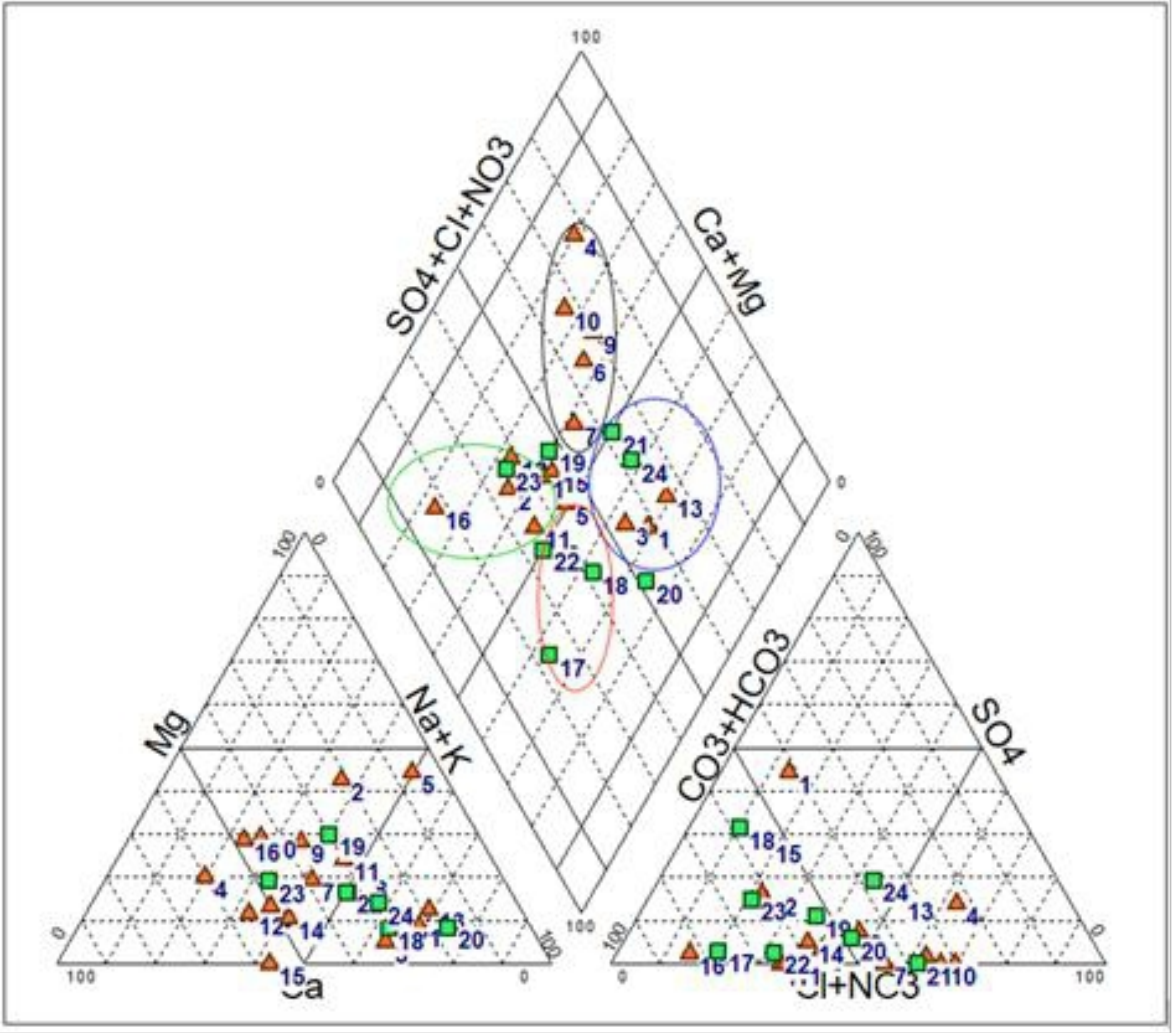

Fig. 5 : Faciès des eaux de la nappe alluviale

Les corrélations significatives $(\geq 60 \%)$ entre les éléments chimiques (tableau 1), sont :

- TDS avec $\mathrm{HCO}_{3}, \mathrm{Na}, \mathrm{Mg}, \mathrm{SO}_{4}$, et $\mathrm{Cl}$, indiquant que la minéralisation totale exprimée par le TDS, est contrôlée par ces principaux ions ;

- $\quad \mathrm{NO}_{3}$ avec $\mathrm{Cl}$, pourrait indiquer la même provenance de ces éléments à savoir à partir de la surface. En effet, les matières organiques décomposées (déjections des animaux, végétation, etc.), les eaux usées et les chlorures provenant de ces éléments ou des eaux de pluies, rejoindraient la nappe alluviale.

- Par ailleurs, la température n'est corrélée à aucun élément, signifiant que son action serait peu significative dans le processus de minéralisation des eaux. 
Tableau 1 : Matrice de corrélation entre les éléments chimiques

\begin{tabular}{|c|c|c|c|c|c|c|c|c|c|c|c|c|}
\hline & $\mathbf{T}^{\circ}$ & pH & $\mathrm{CE}$ & TDS & $\mathrm{HCO}_{3}$ & $\mathrm{Cl}$ & $\mathrm{SO}_{4}$ & $\mathrm{NO}_{3}$ & $\mathrm{Na}$ & $\mathbf{K}$ & $\mathrm{Ca}$ & Mg \\
\hline $\mathbf{T}^{\circ}$ & 1 & & & & & & & & & & & \\
\hline pH & $-0,1$ & 1,0 & & & & & & & & & & \\
\hline $\mathrm{CE}$ & $-0,3$ & 0,8 & 1,0 & & & & & & & & & \\
\hline TDS & $-0,4$ & 0,3 & 0,6 & 1,0 & & & & & & & & \\
\hline $\mathrm{HCO}_{3}$ & $-0,4$ & 0,4 & 0,6 & 0,9 & 1,0 & & & & & & & \\
\hline Cl & $-0,1$ & 0,1 & 0,2 & 0,6 & 0,5 & 1,0 & & & & & & \\
\hline $\mathrm{SO}_{4}$ & $-0,2$ & 0,1 & 0,4 & 0,7 & 0,6 & 0,1 & 1,0 & & & & & \\
\hline $\mathrm{NO}_{3}$ & $-0,2$ & 0,1 & 0,3 & 0,4 & 0,2 & 0,6 & $-0,1$ & 1,0 & & & & \\
\hline $\mathrm{Na}$ & $-0,2$ & 0,3 & 0,5 & 0,9 & 0,8 & 0,4 & 0,8 & 0,2 & 1,0 & & & \\
\hline $\mathbf{K}$ & $-0,4$ & 0,0 & 0,3 & 0,2 & 0,2 & 0,1 & 0,1 & 0,2 & $-0,1$ & 1,0 & & \\
\hline $\mathbf{C a}$ & $-0,5$ & 0,0 & 0,3 & 0,5 & 0,3 & 0,1 & 0,5 & 0,3 & 0,3 & 0,7 & 1,0 & \\
\hline Mg & $-0,2$ & 0,4 & 0,5 & 0,6 & 0,7 & $\mathbf{0 , 8}$ & 0,0 & 0,5 & 0,4 & 0,0 & $-0,1$ & 1,0 \\
\hline
\end{tabular}

Le rapport $\mathrm{Na} / \mathrm{Cl}$ (Fig. 6A), montre une répartition assez équilibrée entre ces deux éléments chimiques notamment au niveau des ouvrages du secteur amont. Ceci indique que ces éléments seraient issus partiellement ou totalement de la même source qui serait les eaux de précipitations. Par contre, au niveau du secteur aval les ions Na prédominent, indiquant que cet élément serait issu de la dissolution des roches évaporitiques.

Le graphique $\mathrm{Ca} / \mathrm{HCO}_{3}$ (Fig. 6B) montre une certaine prédominance des ions bicarbonates sur les ions calcium. Ainsi, les bicarbonates ne seraient pas issus uniquement de la dissolution des carbonates de calcium. Par ailleurs, l'excès des bicarbonates est en accord avec le processus d'évolution d'un système ouvert vers un système fermé.

Le graphique $\mathrm{Ca} / \mathrm{SO}_{4}$ (Fig. 6C) indique une prédominance du Ca sur le SO4. Par conséquent, le calcium ne proviendrait pas d'une dissolution des minéraux sulfatés. Néanmoins, dans le secteur aval, où la nappe alluviale est captée par le puits de Kwakwara, il semble indiquer un certain équilibre entre ces deux éléments. Cela pourrait s'expliquer par la dissolution d'un niveau évaportitiques riche en gypse.

Le diagramme $\mathrm{Cl} / \mathrm{SO}_{4}$ (Fig. 6D) montre en général un excès de $\mathrm{Cl}$ par rapport aux sulfates mettant en évidence que ces deux éléments n'auraient pas la même origine. En effet, ce constat, confirme la provenance du chlore à partir de la surface. 
A

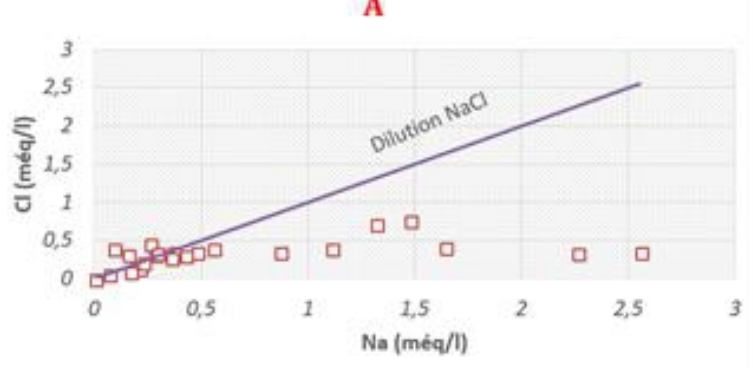

C

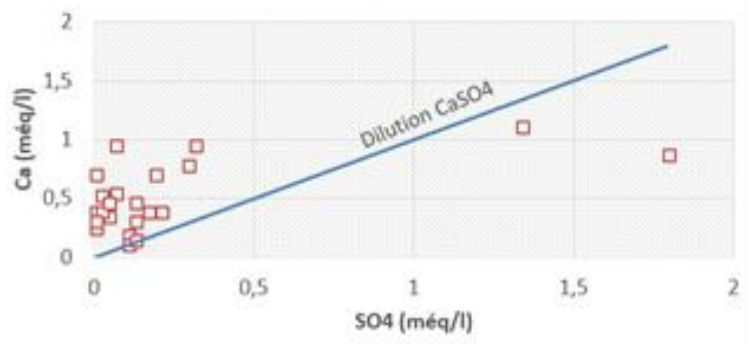

B

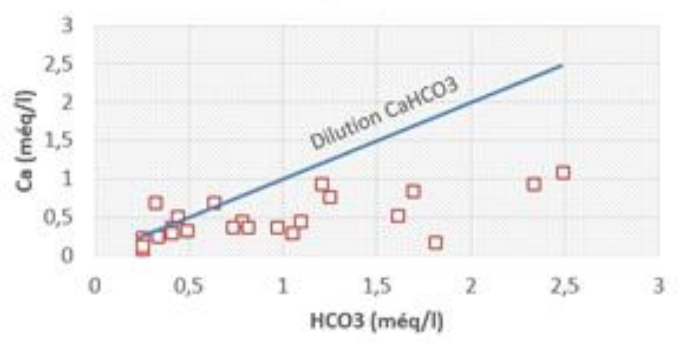

D

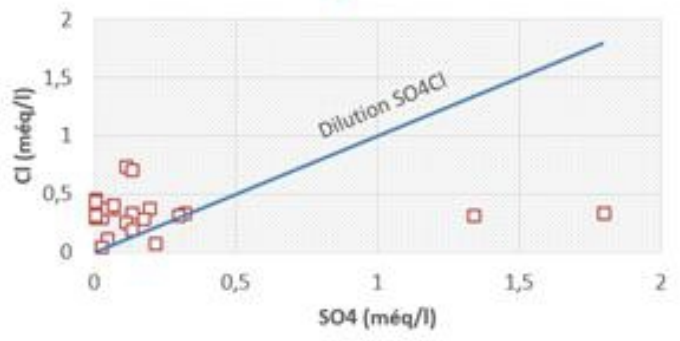

Fig. 6 : Rapports caractéristiques : $\mathrm{Na} / \mathrm{Cl}, \mathrm{Ca} / \mathrm{HCO}_{3}, \mathrm{Ca} / \mathrm{SO}_{4}$, et $\mathrm{Cl} / \mathrm{SO}_{4}$,

L'ACP (fig. 7), montre que les axes factoriels F1 (35,37\%), F2 (19,38\%) et l'axe F3 (11,09\%), représentent environ 66\% de la variance totale (tableau 2).

Tableau 2 : Valeurs propres

\begin{tabular}{llll}
\hline Axes factoriels --> & $\boldsymbol{F 1}$ & $\boldsymbol{F 2}$ & $\boldsymbol{F 3}$ \\
\hline Valeur propre & 4,952 & 2,713 & 1,552 \\
Variabilité (\%) & 35,372 & 19,380 & 11,086 \\
\% cumulé & 35,372 & 54,752 & 65,839
\end{tabular}

L'axe F1 représente négativement la température. Ce paramètre aurait une faible influence sur la minéralisation des eaux. Par ailleurs, il montre un regroupement, des éléments qui sont corrélés entre eux, notamment les bicarbonates, le sodium, les sulfates, le calcium et le potassium confirmant que ces éléments contrôlent la minéralisation et le $\mathrm{pH}$. Cet axe représente la minéralisation totale des eaux. L'axe F2, représente positivement les éléments d'origine anthropiques, notamment les nitrates et les nitrites, mais aussi, les chlorures et le magnésium, c'est l'axe de la pollution des eaux. Il indique également, l'axe de l'infiltration des eaux de précipitations. La projection F1, F3 confirme la bonne corrélation entre la conductivité, le $\mathrm{pH}$ et le $\mathrm{HCO}_{3}$. Par ailleurs, l'ACP confirme que cette minéralisation des eaux de la nappe alluviale évolue avec le $\mathrm{pH}$ et le type de faciès. 


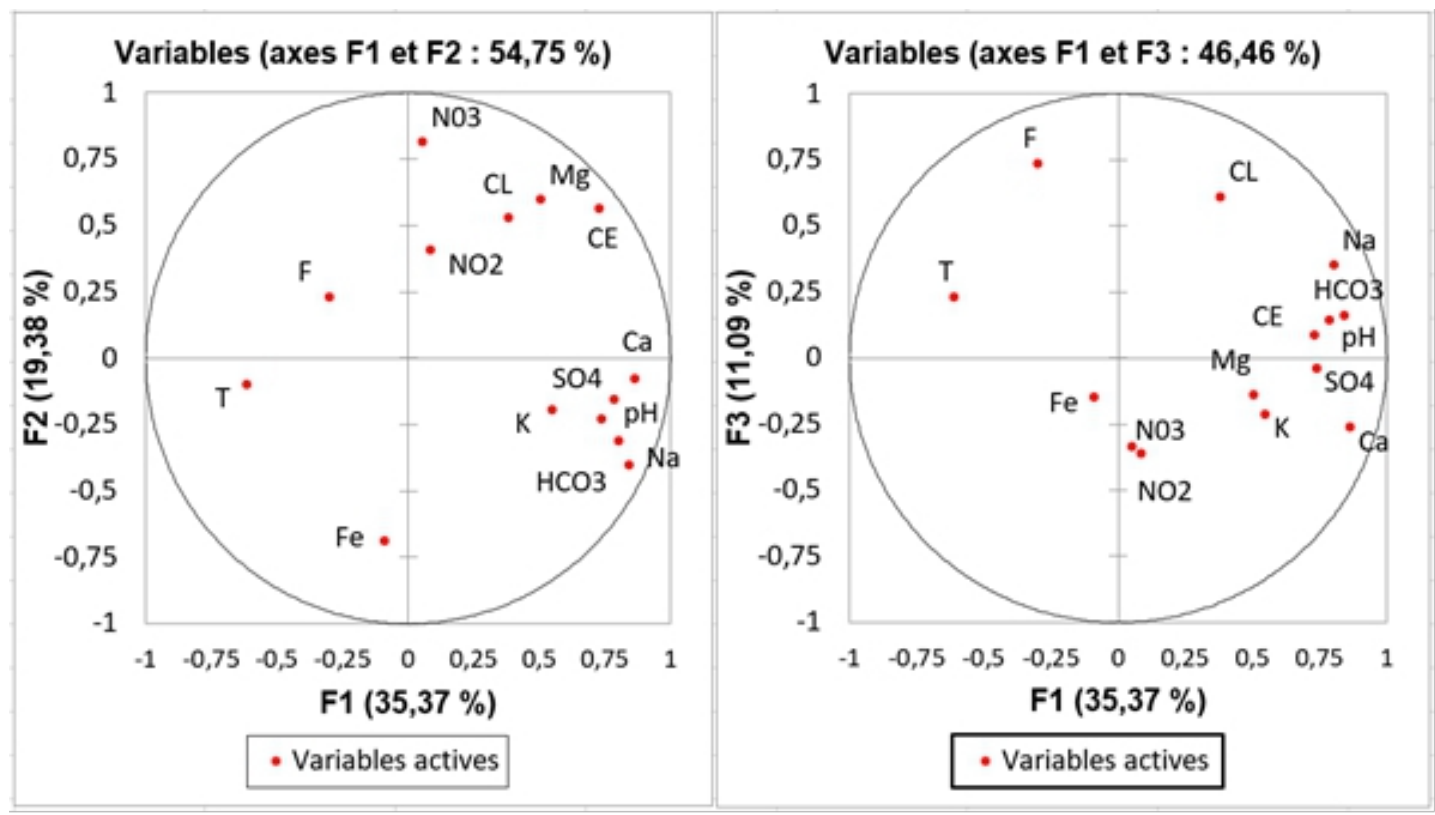

Fig. 7 : ACP- Cercles de corrélation suivant les axes F1-F2 et F1-F3

Les indices de saturation calculés (tableau 3), montrent que pour l'ensemble des sites, ceux-ci sont inférieurs à 0 , indiquant que les eaux de la nappe alluviale sont sous saturées, donc agressives, vis-à-vis de ces minéraux. Ces résultats confirment la faible minéralisation et les basses valeurs de $\mathrm{pH}$. Les eaux du bassin limitrophe de la Korama (Sandao, 2013), ont également des indices de saturations comparables à ceux de la vallée du Goulbi N'Kaba.

Tableau 3 : Indices de saturation des eaux

\begin{tabular}{clccccc}
\hline $\mathbf{N}^{\circ}$ & \multicolumn{1}{c}{ Nom } & Ouvrage & IsCalcite & IsAragonite & IsDolomite & IsGypse \\
\hline 1 & Kwkwara & PC & $-1,14$ & $-1,29$ & $-3,12$ & $-2,3$ \\
2 & Zangon Tachalawa & F/PMH & $-1,71$ & $-1,86$ & $-4,24$ & $-3,14$ \\
3 & Rijia Igdala & PC & $-1,41$ & $-1,56$ & $-4,15$ & $-3,51$ \\
4 & Tagaza & PC & $-1,44$ & $-1,58$ & $-3,71$ & $-3,23$ \\
5 & Mallé Sofoua & PC & $-1,74$ & $-1,88$ & $-3,04$ & $-4,15$ \\
6 & Zangon Gaba & PC & $-2,27$ & $-2,42$ & $-5,26$ & - \\
7 & Mayara & PC & $-1,54$ & $-1,68$ & $-3,75$ & - \\
8 & Dajin Djigo & PC & $-1,67$ & $-1,81$ & $-3,84$ & - \\
9 & Jardin Mayahi & PC & $-1,15$ & $-1,29$ & $-3,41$ & $-2,93$ \\
10 & J. Kahin Kossaou & F/PMH & $-2,46$ & $-2,6$ & $-5,82$ & $-3,95$ \\
11 & Bourlé PC & PC & $-1,99$ & $-2,14$ & $-5,05$ & $-4,19$ \\
12 & Bougouzawa & PC & $\mathbf{- 2 , 0 3}$ & $-2,17$ & - & $-3,45$ \\
13 & Rijia Hardo & PC & $\mathbf{- 1 , 7 7}$ & $-1,92$ & $-4,19$ & $-4,45$
\end{tabular}

L'analyse des teneurs de certains éléments indésirables a permis d'apprécier la qualité des eaux et le niveau de pollution nitratée de la nappe. 
En effet, les teneurs en fer sont comprises entre 0 et $1,05 \mathrm{mg} / \mathrm{l}$, avec une moyenne de $0,13 \mathrm{mg} / \mathrm{l}$. Ces teneurs relativement faibles, semblent indiquer l'absence de source susceptible de pourvoir les eaux de cette nappe en fer.

Les teneurs en fluor varient de 0 à $0,54 \mathrm{mg} / \mathrm{l}$, avec une moyenne de $0,16 \mathrm{mg} / \mathrm{l}$. Ces teneurs sont très faibles et inférieures à la valeur indicative de la norme OMS, 2006, qui est de 1,5 mg/l. Néanmoins, une forte concentration en fluor de 2,59 mg/l (DRHA Maradi, 2014) a été dosée dans les eaux du puits de Malam Kaka. Malgré qu'il soit implanté dans la vallée du Goulbi N'Kaba, cet ouvrage semble capter la nappe du $\mathrm{CI} / \mathrm{CH}$ qui a déjà démontré des fortes concentrations en fluor à Sabon Machi $(5 \mathrm{~km}$ au sud de Malam Kaka), et plus au sud, dans le secteur de Tibiri $(6 \mathrm{mg} / \mathrm{l})$. Ces fortes teneurs en fluor dans les eaux de consommation ont déjà causé des fluoroses dentaires et osseuses (handicapantes) chez les enfants à Tibiri et à Malam Kaka. Le niveau actuel de connaissance du phénomène, ne permet pas d'expliquer l'origine et la distribution spatiale du fluor au niveau des systèmes aquifères. Aussi, il s'avère nécessaire de conduire une étude dans ce sens.

Les teneurs en nitrates varient de 1,32 à 48,4 mg/l, avec une moyenne de $12,79 \mathrm{mg} / \mathrm{l}$ et un écart type de 12,6. Ces valeurs sont relativement faibles et inférieures aux directives de l'OMS, 2006. Cependant, les plus fortes concentrations en nitrates sont observées au niveau des puits pastoraux localisés au centre de la zone d'étude, érigé en aire pastorale. L'origine de ces nitrates serait liée aux activités pastorales. Concernant les nitrites, leurs concentrations varient de 0 à $0,85 \mathrm{mg} / \mathrm{l}$, avec une moyenne de $0,07 \mathrm{mg} / \mathrm{l}$, et un écart type de $0,17 \mathrm{mg} / \mathrm{l}$. Les teneurs en nitrites dépassant les directives de l'OMS, 2006, se trouvent également au centre de la zone d'étude au niveau des puits pastoraux de Tagaza et de Zongon Igdala qui ont respectivement 0,86 $\mathrm{mg} / \mathrm{l}$ et $0,11 \mathrm{mg} / \mathrm{l}$. Les matières fécales des animaux trainées par les puisettes et/ou le retour des eaux usées de surface dans les ouvrages, seraient à l'origine de ces nitrites.

Les indices SAR et les valeurs des conductivités à $25^{\circ} \mathrm{C}$, (tableau 4), représentés sur le diagramme dit de Richards (1954), (Fig. 6), montrent que la majorité des eaux se situe dans la classe S1C1 indiquant que les eaux sont de qualité excellente pour l'irrigation. Par contre, certaines eaux, notamment celles de puits cimentés de Mallé Sofoua, Kwakwara PC et Mayara PC, des secteurs central et amont, se situent dans la classe S1C2.

Tableau 4 : Indices SAR des eaux de la nappe alluviale de la vallée du Goulbi N'Kaba.

\begin{tabular}{lcccccccc}
\hline Localités & $\mathbf{C a}++$ & $\mathbf{M g}++$ & $\mathbf{N a}+$ & $(\mathbf{C a}+\mathbf{M g})$ & $(\mathbf{C a}+\mathbf{M g}) / \mathbf{2}$ & $\sqrt{ }(\mathbf{C a}+\mathbf{M g}) / \mathbf{2}$ & $\mathbf{S A R}$ & Conductivité \\
\hline Kwakwara & 1,12 & 0,324 & 2,26 & 1,44 & 0,72 & 0,85 & $\mathbf{2 , 6 6}$ & 362 \\
Rijia Igdala & 0,4 & 0,405 & 0,42 & 0,81 & 0,40 & 0,63 & $\mathbf{0 , 6 7}$ & 179 \\
Mallé S. & 0,32 & 0,162 & 1,32 & 0,48 & 0,24 & 0,49 & $\mathbf{2 , 6 8}$ & 325 \\
Z. Gaba & 0,32 & 0,162 & 0,30 & 0,48 & 0,24 & 0,49 & $\mathbf{0 , 6 1}$ & 124 \\
Tagaza PC & 0,72 & 0,243 & 0,09 & 0,96 & 0,48 & 0,69 & $\mathbf{0 , 1 3}$ & 206 \\
Maîyara PC & 0,72 & 0,486 & 0,26 & 1,21 & 0,60 & 0,78 & $\mathbf{0 , 3 4}$ & 293 \\
\hline
\end{tabular}




\begin{tabular}{lcccccccc}
\hline H D.Baba & 0,36 & 0,081 & 0,21 & 0,44 & 0,22 & 0,47 & $\mathbf{0 , 4 5}$ & 88 \\
Bougouzawa & 0,4 & 0 & 0,16 & 0,40 & 0,20 & 0,45 & $\mathbf{0 , 3 6}$ & 62 \\
Bourlé PC & 0,4 & 0,243 & 0,07 & 0,64 & 0,32 & 0,57 & $\mathbf{0 , 1 2}$ & 76 \\
Tallalabiya & 0,56 & 0,162 & 1,11 & 0,72 & 0,36 & 0,60 & $\mathbf{1 , 8 5}$ & 110 \\
Dajin Jigo & 0,48 & 0,243 & 0,55 & 0,72 & 0,36 & 0,60 & $\mathbf{0 , 9 2}$ & 184,6 \\
J. Mayahi & 0,8 & 0,324 & 0,29 & 1,12 & 0,56 & 0,75 & $\mathbf{0 , 3 9}$ & 244 \\
Kahin K. & 0,16 & 0,081 & 0,23 & 0,24 & 0,12 & 0,35 & $\mathbf{0 , 6 6}$ & 66,8
\end{tabular}

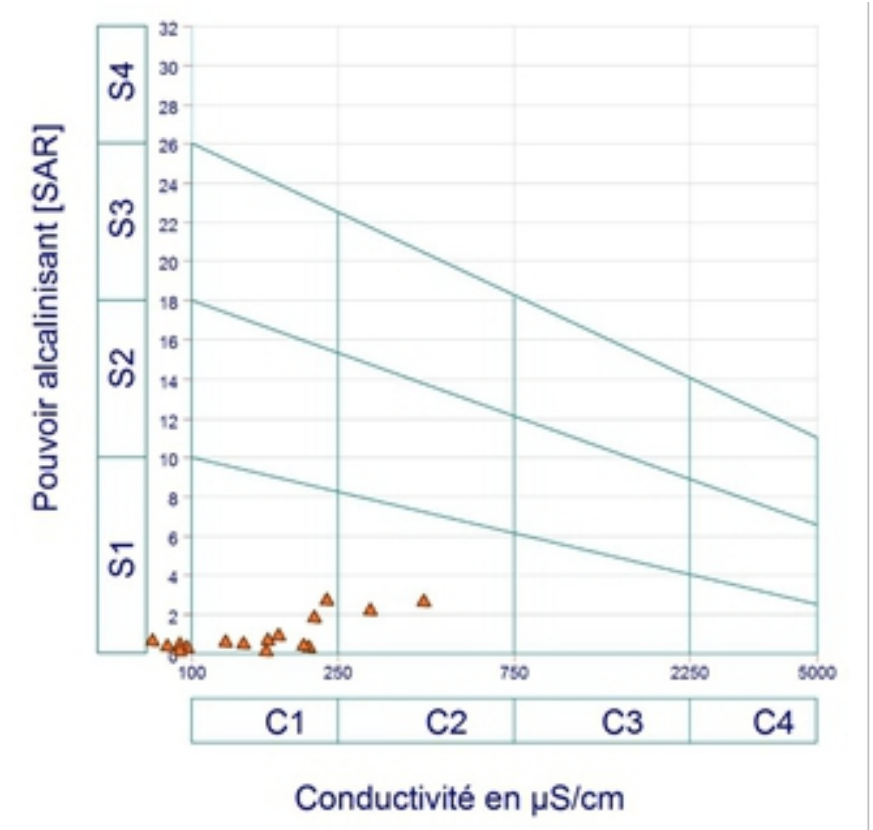

Fig. 8 : Diagramme dit de Richards (1954)

Les valeurs du RSC (tableau 5) sont inférieures à 1,25 et indiquent que ces eaux sont de bonne qualité pour l'irrigation.

Tableau 5 : Valeurs du RSC des eaux

\begin{tabular}{lccccccc}
\hline Sites & $\mathbf{C a}_{++}$ & $\mathbf{M g}_{++}$ & $\mathbf{H C O}_{3-}$ & $\left(\mathbf{C O}_{\mathbf{3}}+\mathbf{H C O}_{\mathbf{3}}\right)$ & $(\mathbf{C a}+\mathbf{M g})$ & $\mathbf{R S C}$ & Classes \\
\hline Kwakwara pc & 1,12 & 0,324 & 2,48 & 2,48 & 1,44 & $\mathbf{1 , 0 4}$ & bonne \\
Rijia Igdala & 0,4 & 0,405 & 0,8 & 0,8 & 0,81 & $\mathbf{- 0 , 0 1}$ & bonne \\
Mallé S. & 0,32 & 0,162 & 1,04 & 1,04 & 0,48 & $\mathbf{0 , 5 6}$ & bonne \\
Zangon Gaba & 0,32 & 0,162 & 0,4 & 0,4 & 0,48 & $\mathbf{- 0 , 0 8}$ & bonne \\
Tagaza PC & 0,72 & 0,243 & 0,31 & 0,31 & 0,96 & $\mathbf{- 0 , 6 5}$ & bonne \\
Maîyara PC & 0,72 & 0,486 & 0,62 & 0,62 & 1,21 & $\mathbf{- 0 , 5 9}$ & bonne \\
H.D Baba & 0,36 & 0,081 & 0,48 & 0,48 & 0,44 & $\mathbf{+ 0 , 0 4}$ & bonne \\
Bougouzawa & 0,4 & 0 & 0,4 & 0,4 & 0,40 & $\mathbf{+ 0 , 0 0}$ & bonne \\
Bourlé PC & 0,4 & 0,243 & 0,72 & 0,72 & 0,64 & $\mathbf{+ 0 , 0 8}$ & bonne \\
Tallalabiya & 0,56 & 0,162 & 1,6 & 1,6 & 0,72 & $\mathbf{+ 0 , 8 8}$ & bonne \\
Dajin Jigo & 0,48 & 0,243 & 1,08 & 1,08 & 0,72 & $\mathbf{+ 0 , 3 6}$ & bonne \\
Jardin Mayahi & 0,8 & 0,324 & 1,24 & 1,24 & 1,12 & $\mathbf{+ 0 , 1 2}$ & bonne \\
Jardin Kahin K. & 0,16 & 0,081 & 0,24 & 0,24 & 0,24 & $\mathbf{+ 0 , 0 0}$ & bonne
\end{tabular}


Le diagramme de Wilcox (Fig.9), montre que toutes les eaux appartiennent à la catégorie des eaux de qualité excellente pour leur aptitude à l'irrigation.

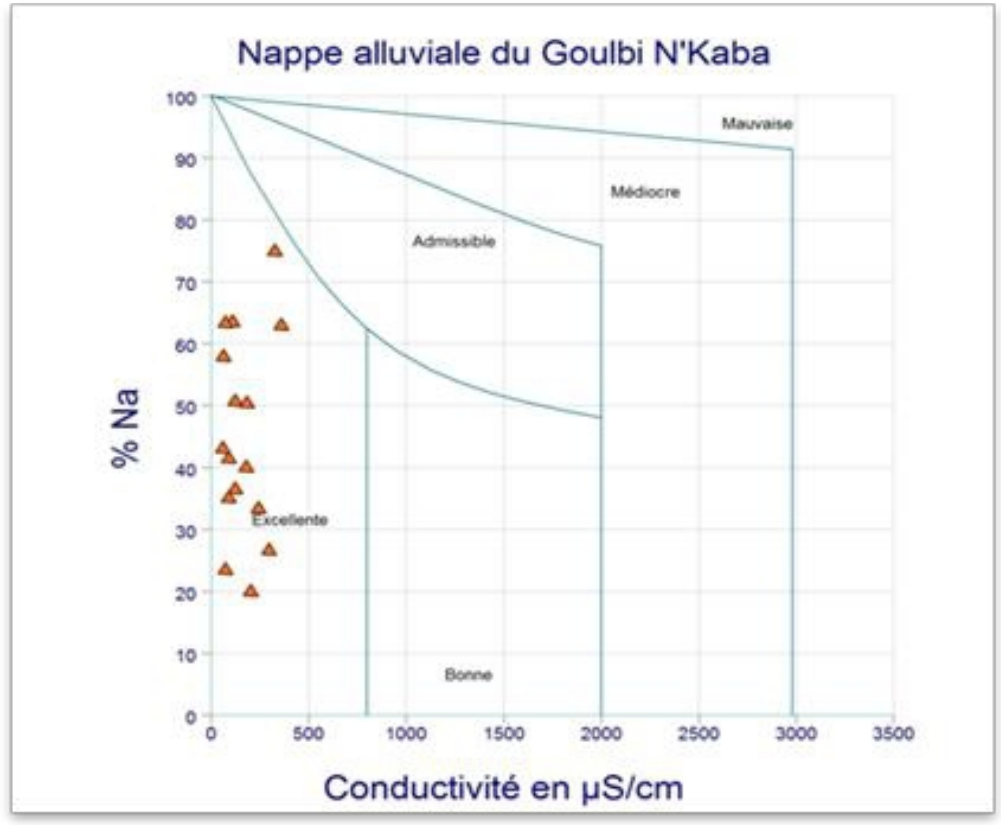

Fig. 9 : Diagramme de Wilcox

\section{Conclusion}

Les quelques résultats des études des paramètres physico-chimiques, exposés dans cet article, montrent que les eaux de la nappe alluviale ont un $\mathrm{pH}$ légèrement acide et sont faiblement minéralisées. Ces paramètres croissent de l'amont vers l'aval où le système tend à être fermé.

Les indices de saturations négatifs, confirment la faible minéralisation des eaux qui sont par conséquent agressives vis-à-vis des principaux éléments chimiques.

L'ACP a montré que la minéralisation est essentiellement contrôlée par les bicarbonates, le sodium, le calcium et les sulfates. Elle montre également, que la pollution nitratée relevée dans le secteur central est d'origine anthropique, liée à la pratique d'élevage notamment l'abreuvement autour des puits cimentés.

Les teneurs excessives en fluor relevées au niveau des ouvrages du secteur de Malam Kaka, au centre de la zone d'étude, semblent indiquer que dans ce secteur, la nappe alluviale serait confondue avec celles du $\mathrm{CI} / \mathrm{CH}$.

\section{Remerciements}

Ce travail a été rendu possible grâce au financement du bureau de la coopération Suisse au Niger à travers le Programme d'Hydraulique Rurale, 
Appui au Secteur Eau et Assainissement (PHRASEA) et aux appuis de l'ONG HELVETAS SWISS INTERCOOPERATION et des Directions Régionales de l'Hydraulique et de l'Assainissement de Maradi et de Zinder. Que ces structures et leurs personnels trouvent ici, nos sincères remerciements.

\section{References:}

1. BCEOM/CEH SIDI, Programme d'hydraulique villageoise dans la région de Maradi - Départements d'Aguié, Mayahi et Tessaoua, résultats des analyses physico-chimiques, (2006) annexes 7, 9 et 11

2. BEFAC-REP, Schéma régional d'aménagement du territoire (SRAT) de Maradi (2009), 13-16

3. BOUBACAR, Impacts des sous-projets du PIP2 (Projet de Promotion de l'Irrigation Privée) dans la région de Maradi ; Mémoire de master 2, 2IE Ouagadougou (2007), 62 pages

4. BOUKARI I. Ousmane, Impact de l'irrigation et de la variabilité climatique sur la nappe alluviale du Goulbi Maradi : cas du périmètre irrigué de Djiratawa, UAM, mémoire de master en hydrogéologie (2014)

5. CABINET LABARAN, prospections géophysiques : réalisation de 23 sondages électriques au niveau de la vallée du Goulbi N'Kaba (2016), 62 pages

6. DRHA Maradi, Rapport succinct sur la qualité de l'eau du village de Malam Kaka dans la commune de Sabon Machi, département de Dakoro (2014)

7. GREIGERT, Atlas des eaux souterraines du Niger, Etat des connaissances (1968), Tome 1 - Fascicules 1,3 et 8

8. INS, $4^{\text {ème }}$ recensement général de la population et de l'habitat du Niger (2012), structure région de Maradi

9. MAHE G., OLIVRY J.C.\& SERVAT E., sensibilité des cours d'eau ouest africains aux changements climatiques et environnementaux : extrêmes et paradoxes, IAHS Publ. (2005), 169-177

10. MHE, Projet d'aménagement de la vallée du Goulbi N'Kaba Département de Maradi, (1993) 78 pages

11. OUSMANE B. - Contribution à l'étude hydrogéologique de socle du Sahel: l'hydrogéologie de Damagaram Occidental (environs de Zinder Niger), Thèse de 3ième cycle USTL, Montpellier (1978) ;

12. PIP2, Projet de Promotion de l'Irrigation Privée au Niger, Rapport annuel d'activités (2006), 157 pages

13. SOGETHA, le Goulbi N'Kaba et ses affluents : reconnaissance hydrogéologique, annexes, figures et cartes, Ministère de l'Economie Rurale du Niger (1964), 195 pages 
14. SANDAO, Etudes Hydrodynamique, Hydro-chimique et Isotopique des eaux souterraines du bassin versant de la Korama / Sud Zinder, Niger: Impacts de la variabilité climatique et des activités anthropiques, thèse de doctorat, Université Abdou Moumouni de Niamey (2013), 178 pages

15. SANDAO, BABAYE, OUSMANE, MICHELOT, Qualité des eaux et vulnérabilité à la pollution des nappes souterraines dans le bassin de la Korama au Niger, Afrique SCIENCE (2015) 364 - 379. 